\title{
Transient response of a polymer electrolyte membrane fuel cell subjected to modulating cell voltage
}

\author{
Shamsuddin Noorani ${ }^{1}$ and Tariq Shamim ${ }^{2, *, \dagger}$ \\ ${ }^{1}$ Department of Mechanical Engineering, The University of Michigan-Dearborn, Dearborn, MI 48128-2406 \\ ${ }^{2}$ Mechanical Engineering, Masdar Institute of Science and Technology, Abu Dhabi, UAE
}

\section{SUMMARY}

This paper presents a computational investigation of the effect of time-varying modulating conditions on a polymer electrolyte membrane fuel cell. The focus is on developing a better understanding of the fuel cell's water balance under transient conditions, which is critical in improving the fuel cell design. The study employs a macroscopic single-fuel cell-based, onedimensional, isothermal model. The model does not rely on the non-physical assumption of the uptake curve equilibrium between the pore vapor and ionomer water in the catalyst layers. Instead, the transition between the two phases is modeled as a finite-rate equilibration process. The modulating conditions are simulated by forcing the temporal variations in fuel cell voltage. The results show that cell voltage modulations cause a departure in the cell behavior from its steady behavior, and the finite-rate equilibration between the catalyst vapor and liquid water can be a factor in determining the cell response. The cell response is also affected by the modulating frequency and amplitude. The peak cell response is observed at low frequencies. Copyright (C) 2011 John Wiley \& Sons, Ltd.

\section{KEY WORDS}

fuel cell; water transport; dynamic behavior; numerical simulations

\section{Correspondence}

*Tariq Shamim, Professor of Mechanical Engineering, Masdar Institute of Science \& Technology, Abu Dhabi, UAE.

†E-mail: tshamim@masdar.ac.ae

Received 15 July 2011; Accepted 9 August 2011

\section{INTRODUCTION}

Fuel cell-based propulsion system for vehicles is gaining significant interest due to a general awareness to reduce air pollution and carbon dioxide emissions and the need to reduce dependence on fossil-based fuels. However, to compete with the proven internal combustion engine-based propulsion, a fuel cell-based propulsion system for transportation sector must show significant advantages in terms of overall efficiency, weight, packaging, safety, cost, and meet various strict requirements. A key requirement is its ability to operate under highly transient conditions (e.g., during start-up, acceleration, and deceleration) with stable performance. Hence, an improved understanding of fuel cell dynamic behavior is critical to its design and control.

Water management and distributions inside the cell are another critical issue in polymer electrolyte membrane (PEM) fuel cell design [1-3]. The optimum performance of fuel cell requires careful balancing between membrane hydration requirement and the electrode flooding avoidance. Due to its importance, it is one of the most widely studied issues in PEM fuel cell area. The past studies have generated a good understanding of water distribution inside the cell especially under steady state conditions as discussed in Wang [1]. However, there are relatively fewer studies which investigated the effect of transient conditions on water distribution inside the cell [4-10]. It should be noted that water transport, particularly, membrane water transport due to slow membrane uptake, is a dominant mechanism in controlling the fuel cell dynamic behavior [11-13]. Furthermore, the humidification requirement of the fuel cell varies with the changes in operating conditions. Hence, understanding of water transport during transient conditions is critical in developing methodologies for optimum fuel cell performance under transient conditions.

Using a three-dimensional model, Wang and Wang [4,5] studied the effect of step increase in current density, cell voltage, and humidity on the PEM fuel cell dynamic performance. They estimated time constant for membrane hydration and showed that the time for fuel cells to reach steady state was in the order of $10 \mathrm{~s}$ due to the effect of water accumulation in the membrane. Song et al. [6] investigated the transient dynamics of water transport in the cathode gas diffusion layer of PEM fuel cells using a 
one-dimensional, two-phase, non-isothermal transient model. They elucidated the effects of four parameters, namely the liquid water saturation at the interface of the gas diffusion layer and flow channels, the proportion of liquid water to all of the water at the interface of the cathode catalyst layer and the gas diffusion layer, the current density, and the contact or wetting angle, on the transient distribution of liquid water saturation in the cathode gas diffusion layer.

Cho et al. [7] performed an experimental study on the transient response of a real automotive-size PEM fuel cell (effective area of $330 \mathrm{~cm}^{2}$ ) under various operating and flooding conditions. The study observed undershoot and overshoot behavior of voltage and time delay when the fuel cell is subjected to step changes in current. Shimpalee et al. [8] determined that water phase change and non-isothermal effects are important during step changes in voltage. Didierjean et al. [9] also found that the voltage response depends on the characteristic times of water transport in the membrane. Vorobev et al. [10] investigated the effect of finite-time equilibration between vapor and membranephase liquid water within the catalyst layers on the fuel cell transient response to step changes in voltage and external humidity conditions.

In many past studies, the transient conditions were generated by considering step changes in fuel cell current or voltage. A step change is an important transient type; however, a fuel cell is subjected to various types of transients during its operation. Hence, the investigation of other types of transients is important in elucidating the dynamic behavior of fuel cell. In the present study, the transient conditions were simulated by considering time-varying modulating cell voltages. It investigates the effect of modulating cell voltage on the cell's liquid and vapor concentrations by employing a mathematical model. The unique feature of the model, presented in Vorobev et al. [10], is that it does not rely on the non-physical assumption of the uptake curve equilibrium between the pore vapor and ionomer water in the catalyst layers. Instead, the transition between the two phases is modeled as a finite-rate equilibration process as proposed by Berg et al. [14,15].

\section{MATHEMATICAL FORMULATION}

A schematic of the fuel cell considered in this paper is illustrated in Figure 1. The cell consists of porous anode and cathode diffusive layers, membrane, and anode and cathode catalyst layers. The model does not include the anode and cathode gas channels, so the boundary conditions are applied directly at the borders of the anode and cathode. Considering the focus of the present work, a onedimensional, transient approach is taken, with all fields being functions of the coordinate $x$ across the cell and time $t$. The model assumes: low humidity operation; no liquid droplets in the diffusion layers; no condensation occurs in the diffusive layers; the membrane (including the membrane phase of the catalyst) is filled with liquid water; phase transition between liquid water and vapor occurs at the surface of the membrane phase; and the operating temperature of the fuel cell is constant. Using these assumptions and notations listed elsewhere [10], the governing equations for the five components of fuel cell may be written as shown in Table I.

The novelty of the model is in the description of the transport and phase transformation of water within the catalyst layers. Compared to other existing models, the present model does not rely on the non-physical assumption of the uptake curve equilibrium between the pore vapor and ionomer water in the catalyst layers. Instead, the transition between the two phases is modeled as a finite-rate equilibration process by employing a phenomenological parameter $\gamma$, which has the physical meaning of the nondimensional reciprocal equilibration time and is defined as $\gamma \equiv \tau_{\mathrm{g}} / \tau_{\gamma}$, where $\tau_{\gamma}$ is the typical relaxation (equilibration) time and $\tau_{\mathrm{g}}$ is the typical gas diffusion time of the

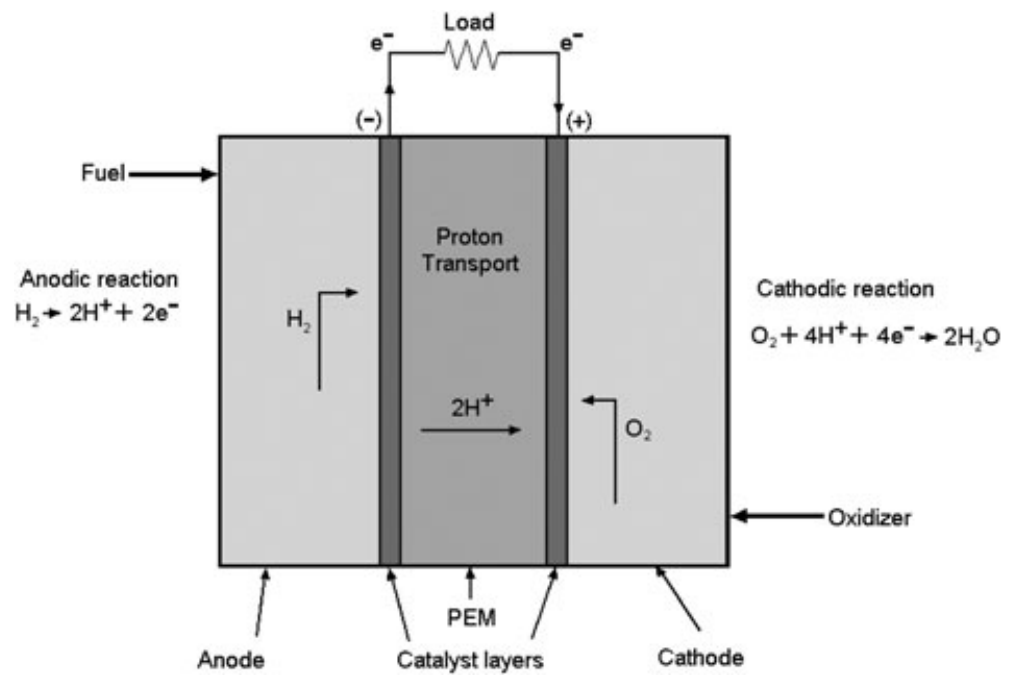

Figure 1. Schematic of a fuel cell. 
Table I. Governing equations for the fuel cell.

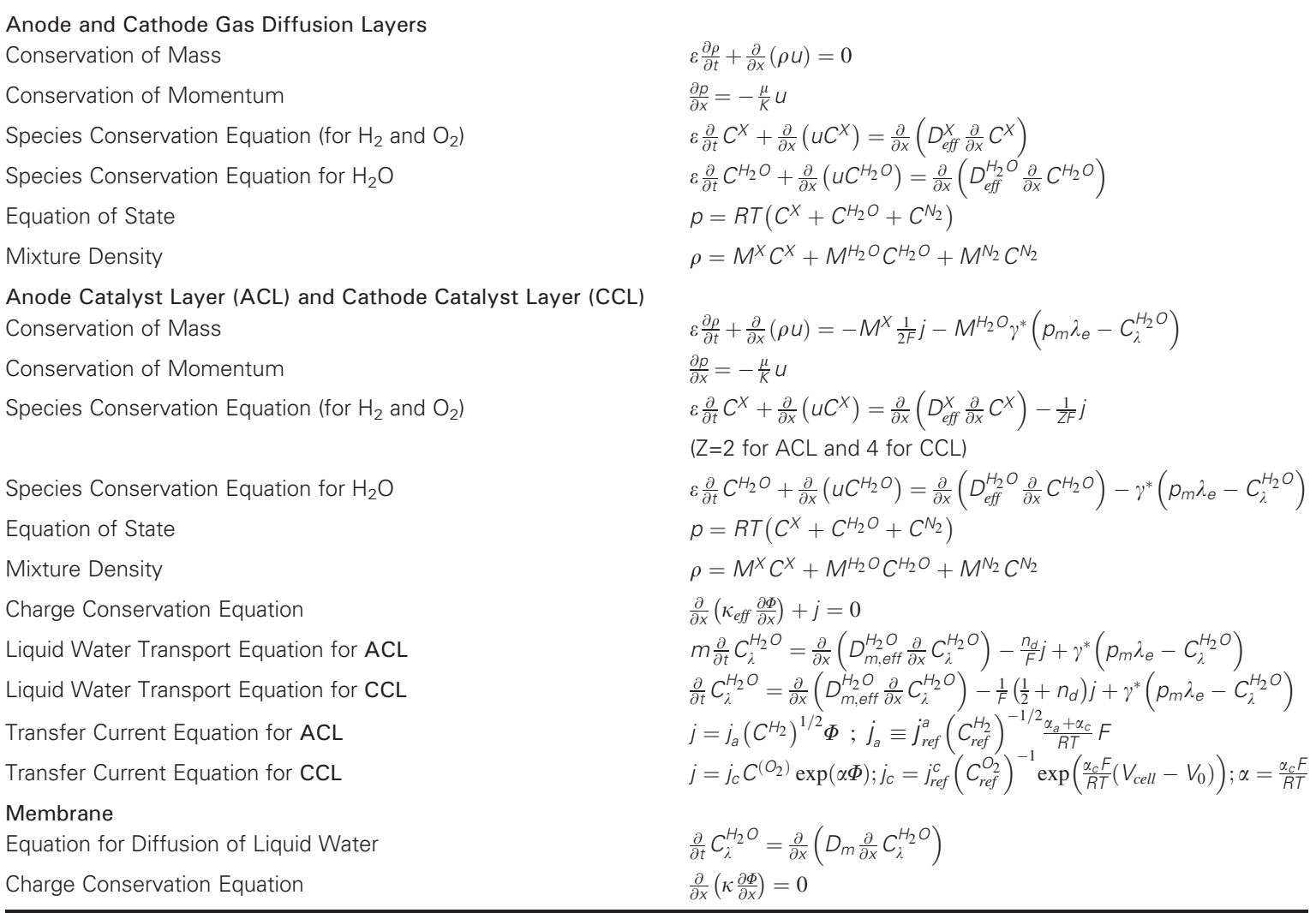

$\mathrm{X}=\mathrm{H}_{2}$ for anode and ACL; $\mathrm{X}=\mathrm{O}_{2}$ for cathode and CCL.

reactant gases through anode and cathode. The relaxation time primarily depends on the volume fraction of the ionomer in the catalyst layer and the area of the contact surface. The upper bound of this time scale can be estimated as $\tau_{\gamma}^{\max }=\left(m^{1 / 3} L_{c}\right)^{2} / D_{m, e f f} \sim 0.5 \mathrm{~s}\left(L_{c}\right.$ is the thickness of the catalyst layer, $m$ is the volume fraction of ionomer in the catalyst layer, and $D_{m, e f f}$ is the effective diffusivity coefficient of the ionomer water). The diffusion time is estimated as $L^{2} / D_{\text {eff }}{ }^{H_{2}}$ ( $L$ is the total thickness of the cell, and $D_{\text {eff }}{ }^{H_{2}}$ is the effective diffusivity of hydrogen), and it ranges from $0.001 \mathrm{~s}$ to $0.05 \mathrm{~s}$. The parameter $\gamma$ is varied between 100 (almost immediate equilibration) and 0.1 (very slow equilibration).

Table II lists the physical quantities used in simulations, which were taken from Wang and Wang [16]. All the boundary conditions used in simulations are similar to those listed in Vorobev et al. [10]. The system of governing equations was solved by the finite-difference method. Second-order central discretization formulas were used for spatial discretization, and Runge-Kutta method of second order was used for the temporal integration. The spatial node size varied in each sub-domain ranging from $1.5 \times 10^{-5} \mathrm{~m}$ for the anode/cathode, $2.55 \times 10^{-6} \mathrm{~m}$ for the membrane and $5 \times 10^{-7} \mathrm{~m}$ for the catalyst layers. The time step was constant and equal to $4 \times 10^{-7} \mathrm{~s}$. The grid insensitivity of results was ensured by performing a sensitivity study. Details of the solution procedure are described elsewhere [10].

The initial conditions were described by specifying the uniform distributions of concentration of hydrogen in the anode and of oxygen in the cathode, of vapor and nitrogen in both parts, as well as uniform pressure throughout the entire fuel cell. The water content in the membrane was assumed to be initially in equilibrium with the vapor concentration. The cell was assumed to be fed by pure hydrogen at the anode side and by air on the cathode side.

The modulating cell voltage conditions were simulated by using the following sinusoidal input:

$$
V_{\text {cell }}(t)=V_{\text {cell-mean }} \times(1+A \times \sin (2 \pi f t))
$$

where $A$ is amplitude, and $f$ is frequency of the modulations. It is worth noting that the real transients are quite random and chaotic, but the simulations consider a mathematically smooth sinusoidal function. This modulation function was selected since it provides many advantages, such as better understanding of the fuel cell response to modulation in terms of its amplitude and frequency, and ease in comparison with experimental and analytical results. For these reasons, many past studies used sinusoidal changes to simulate transient conditions [17-19]. 


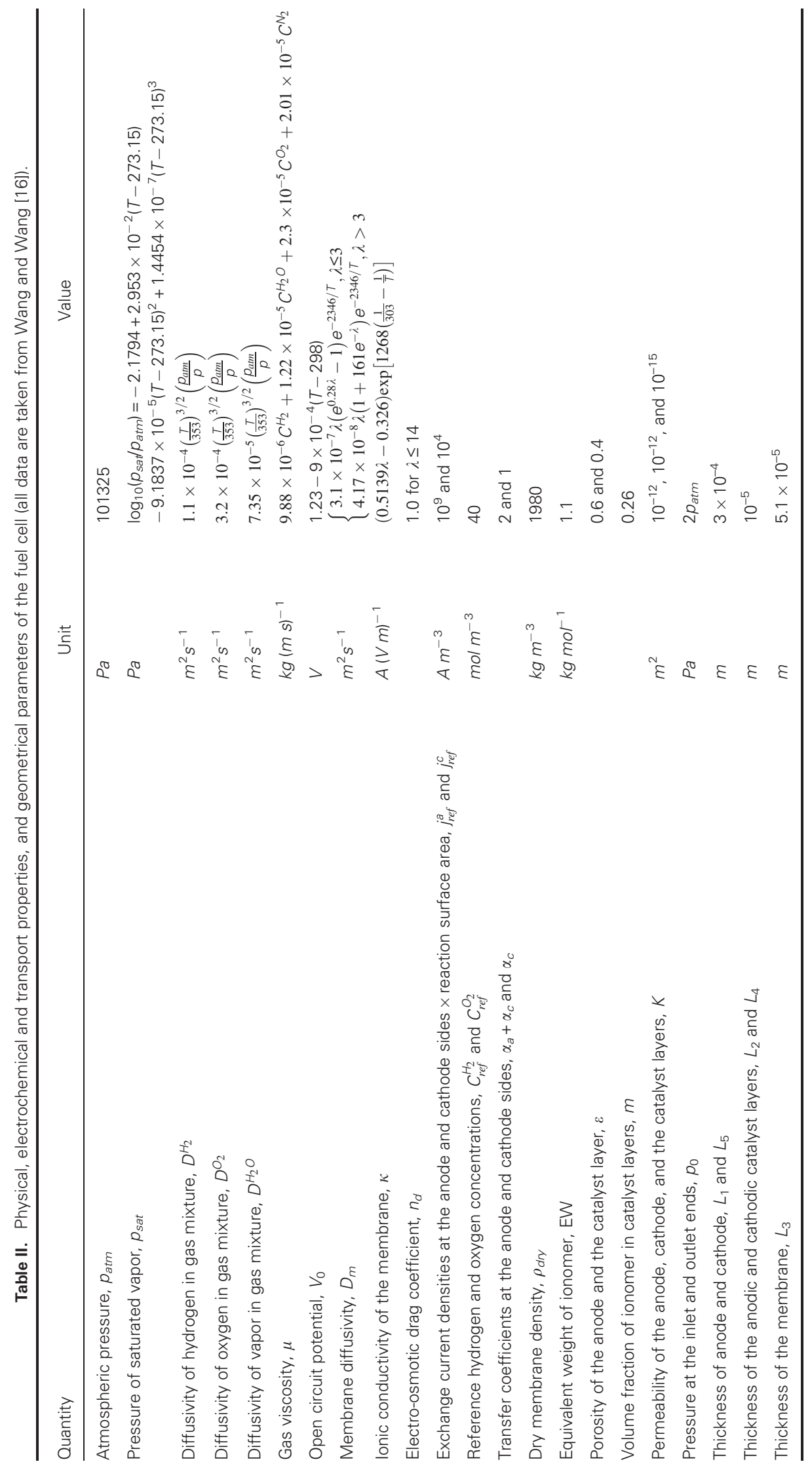


However, this function makes the simulated conditions different from the realistic modulations.

\section{RESULTS AND DISCUSSION}

The numerical model was validated by comparing the polarization curve of the present model with the experimental results of Liu et al. [20], as shown in Figure 2. The figure shows a good agreement between the simulation and experimental results, particularly at low current density values. At high current density values, there is relatively larger difference between the simulation and experimental results. This discrepancy was also observed by Liu et al. [20], who attributed it to the influence of the rib. The numerical model was also validated by comparing with the results of a three-dimensional model of Wang and Wang [16] as reported in our previous paper [10]. The validation results showed the suitability of the present model in simulating the performance of fuel cell. The fuel cell geometry and operating conditions are similar to that reported in our previous paper [10].

The influence of modulating conditions on the fuel cell dynamic behavior was investigated by considering a steady operating fuel cell suddenly subjected to sinusoidal variations of cell voltage. During these oscillations, other inlet operating conditions remained unchanged. Figures 3-5 show the results of the imposed sinusoidal modulation in cell voltage, initially set at $0.7 \mathrm{~V}$, with a frequency of $1 \mathrm{~Hz}$ and amplitude of $10 \%$, and $\gamma=100$. The modulation causes the cell voltage to vary between $0.63 \mathrm{~V}$ and $0.77 \mathrm{~V}$ during each modulation time period.

Figure 3 shows the average liquid water content $\langle\lambda\rangle$ (which is defined as the number of water molecules per sulfonic-acid group) in the membrane and the anodic and cathodic catalyst layers, which respond sinusoidally to the sinusoidal imposed cell voltage modulation. Similar to the steady-state behavior [10], $\langle\lambda\rangle$ in the anodic catalyst layer increases with an increase of cell voltage. Its values

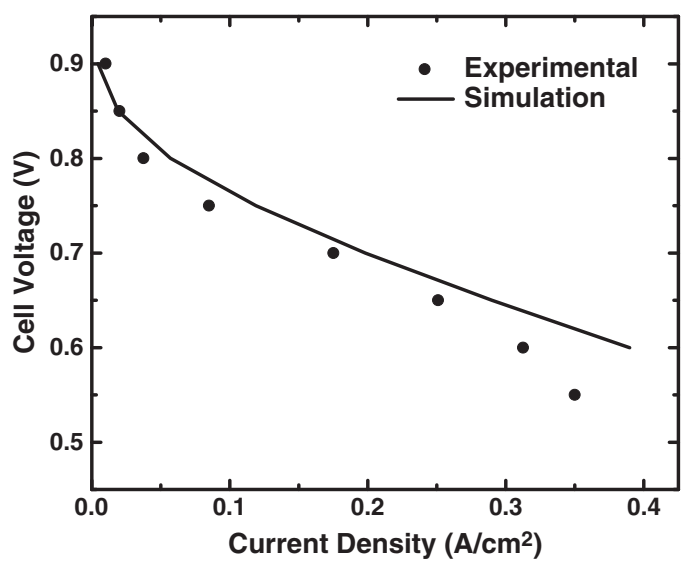

Figure 2. Experimental validation of the model: Comparison of polarization curve with the experimental results of Liu et al. [20].

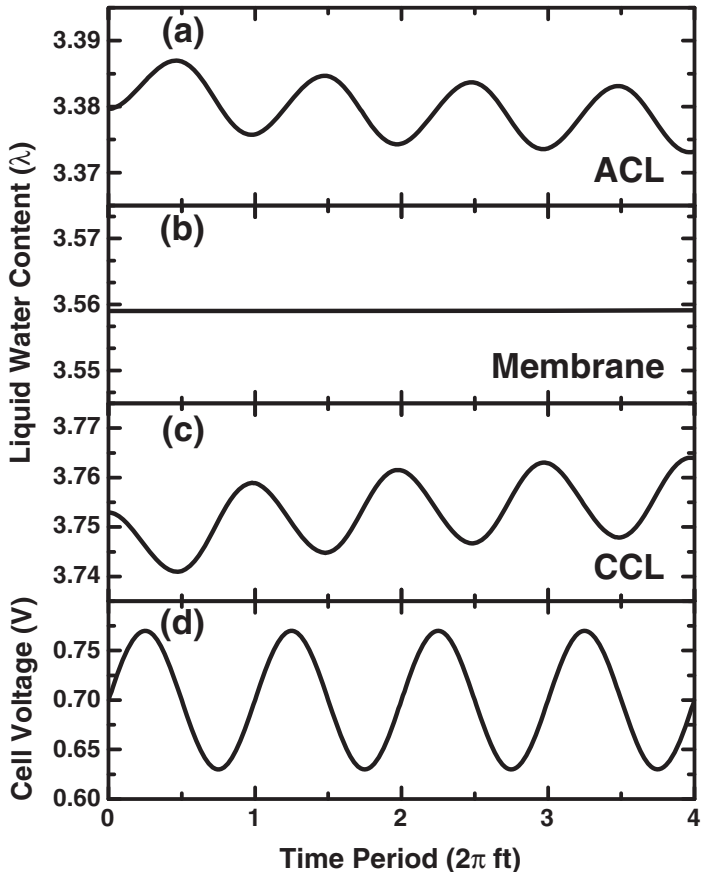

Figure 3. Effect of the cell voltage modulations on the average liquid water content (modulation frequency $=1 \mathrm{~Hz}$, modulation amplitude $=10 \%$, relaxation parameter $(\gamma)=100$ ): (a) anodic catalyst layer; (b) cell membrane; (c) cathodic catalyst layer; (d) imposed cell voltage modulation.

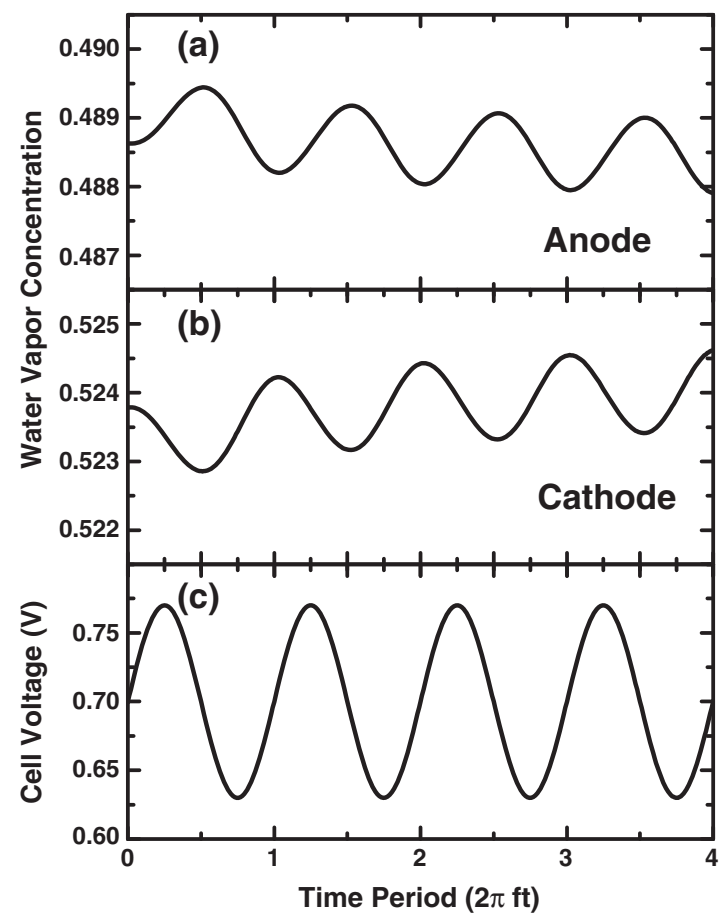

Figure 4. Effect of the cell voltage modulations on the water vapor content (modulation frequency $=1 \mathrm{~Hz}$, modulation amplitude $=10 \%$, relaxation parameter $(\gamma)=100)$ : (a) anode; (b) cathode; (c) imposed cell voltage modulation. 


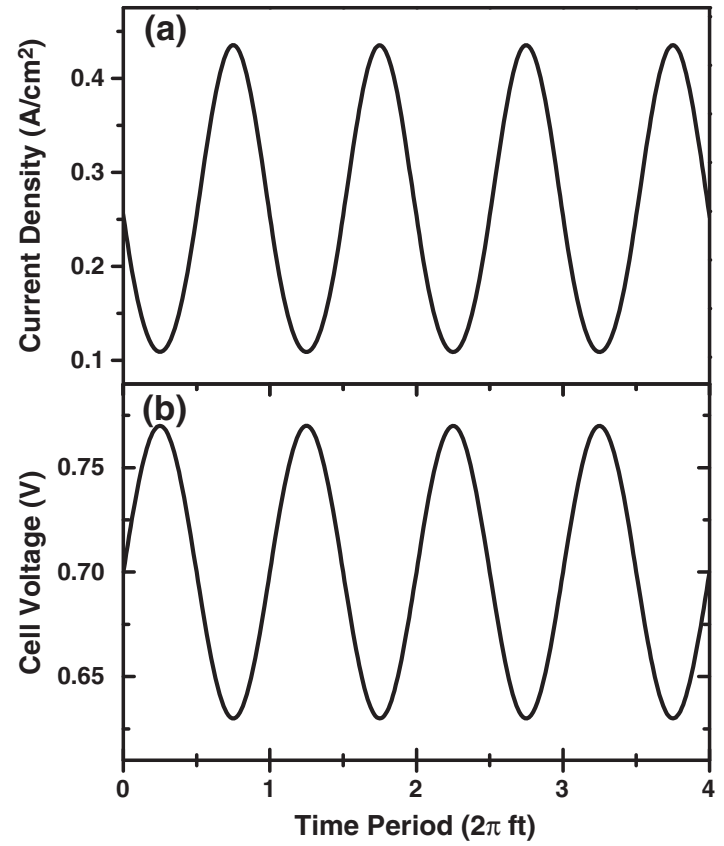

Figure 5. Effect of cell voltage modulations on the current density (modulation frequency $=1 \mathrm{~Hz}$, modulation amplitude $=10 \%$, relaxation parameter $(\gamma)=100$ ): (a) current density; (b) imposed cell voltage modulation.

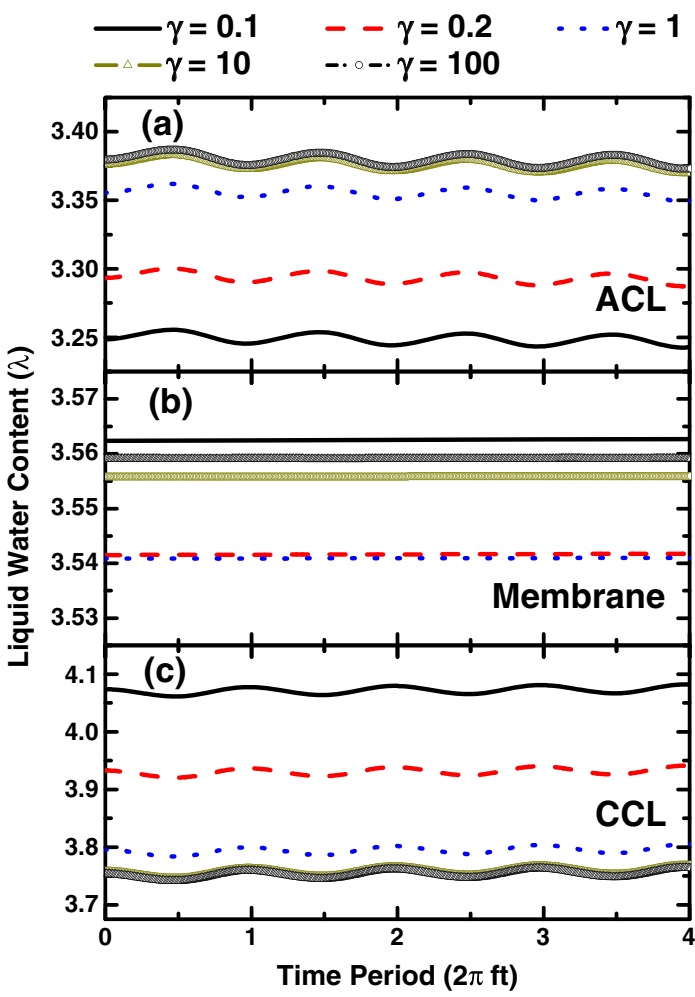

Figure 6. Effect of relaxation parameter $(\gamma)$ on the cell average liquid water content response to modulations in cell voltage (modulation frequency $=1 \mathrm{~Hz}$, modulation amplitude $=10 \%$ ): (a) anodic catalyst layer; (b) cell membrane; (c) cathodic catalyst layer.

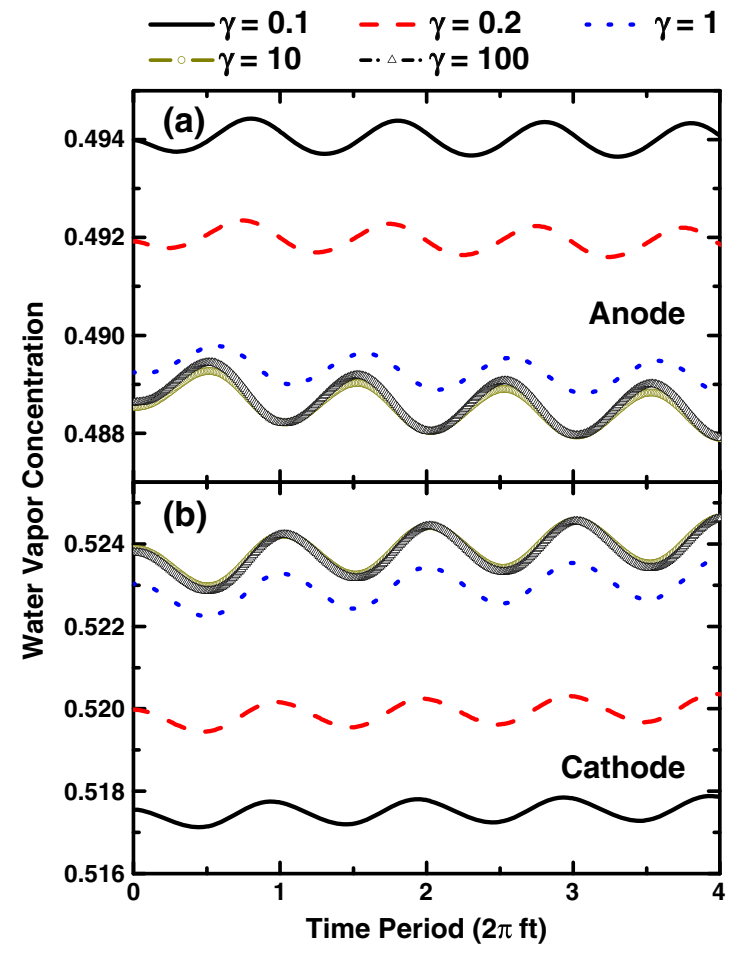

Figure 7. Effect of relaxation parameter $(\gamma)$ on the cell water vapor content response to modulations in cell voltage (modulation frequency $=1 \mathrm{~Hz}$, amplitude $=10 \%$ ): (a) anode; (b) cathode

follow the sinusoidal pattern of the modulating cell voltage. $\langle\lambda\rangle$ attains the maximum and minimum values corresponding to the peak and trough values of the cell voltage. The response amplitude (defined as the difference of peak and trough values divided by the steady-state value corresponding to the mean cell voltage) is small $(0.3 \%)$. With the passage of time, the mean value, around which $\langle\lambda\rangle$ oscillates, undergoes a slight decrease. The maximum and minimum values of $\langle\lambda\rangle$ for modulating conditions are different from the steady-state values of $\langle\lambda\rangle$ corresponding to the the peak and trough values of the cell voltage. The difference of the maximum and minimum values of $\langle\lambda\rangle$ for the modulating condition is $8 \%$ of the corresponding difference for the steady-state case.

The response of $\langle\lambda\rangle$ in the cathodic catalyst layer is similar to that of $\langle\lambda\rangle$ in the anodic catalyst layer except that its amplitude is larger $(0.43 \%)$. Furthermore, the modulation brings about a slight increase of the mean value around which $\langle\lambda\rangle$ in the cathodic catalyst layer oscillates. In the cathodic catalyst layer, the difference of the maximum and minimum values of $\langle\lambda\rangle$ for the modulating condition is only $4 \%$ of the corresponding difference for the steady-state case. The response of $\langle\lambda\rangle$ in the membrane is very similar to those of $\langle\lambda\rangle$ in the catalyst layers except that its amplitude is extremely small.

Figure 4 shows the effect of modulating cell voltage on the average vapor concentrations (expressed in nondimensional form as $R T C^{\mathrm{H}_{2} \mathrm{O}} / p_{\text {sat }}$ ) in anode and cathode, 


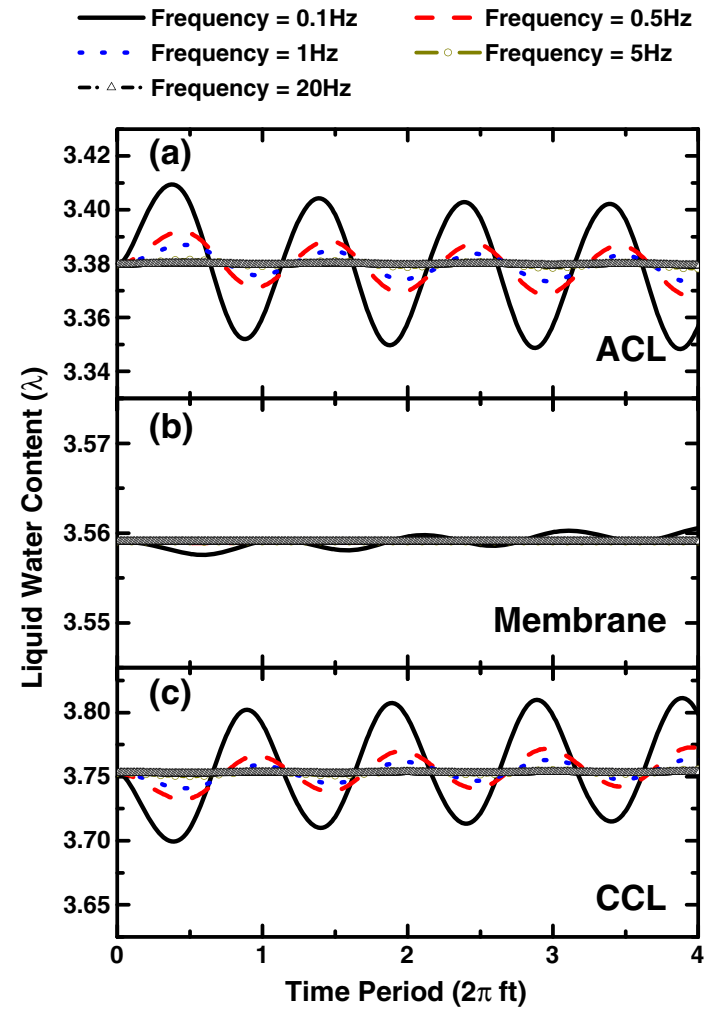

Figure 8. Effect of modulation frequency on the cell average liquid water content response to modulations in cell voltage (modulation amplitude $=10 \%$; relaxation parameter $(\gamma)=100$ ): (a) anodic cata-

lyst layer; (b) cell membrane; (c) cathodic catalyst layer.

which also respond sinusoidally. The response amplitudes of vapor concentration are also small $(0.23 \%)$. The differences of the maximum and minimum values of vapor concentrations in anode and cathode for the modulating condition are $7.6 \%$ and $4 \%$, respectively, of the corresponding differences for the steady-state case. The mean value, around which vapor concentration oscillates, exhibits a slightly decreasing trend in anode and a slightly increasing trend in cathode. This trend disappears, and the mean value becomes constant after a large number of time periods.

The current density responds strongly (with a high amplitude) to the imposed voltage modulations (see Figure 5). The response is sinusoidal to the sinusoidal imposed modulations; however, the response is $180^{\circ}$ out of phase. This was expected since the current density decreases with an increase of voltage in according to the current-voltage characteristics fo the fuel cell.

Figures 6 and 7 show the effect of relaxation parameter $(\gamma)$ on the fuel cell water distribution during transient conditions. These results were obtained by considering sinusoidal modulation in cell voltage with a frequency of $1 \mathrm{~Hz}$ and amplitude of $10 \%$. Results for five different values of $\gamma$ (i.e., $\gamma=0.1,0.2,1,10$, and 100) are shown. Figure 6 depicts that the initial and, therefore, mean values of $\langle\lambda\rangle$ are different for different values of $\gamma$. This is in

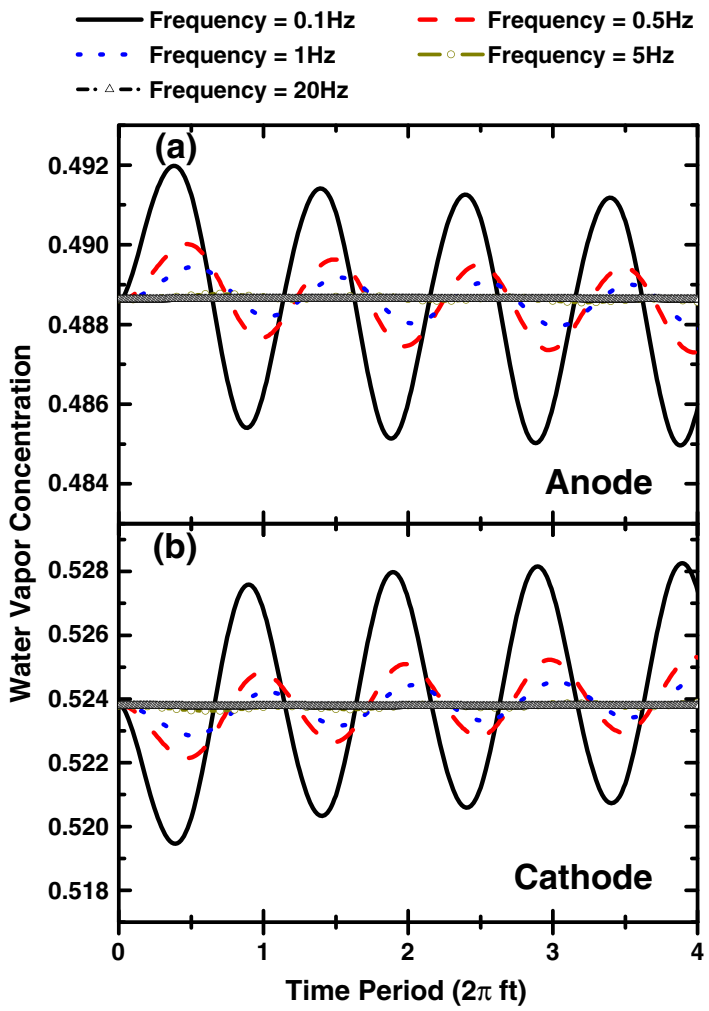

Figure 9. Effect of modulation frequency on the cell water vapor content response to modulations in cell voltage (modulation amplitude $=10 \%$; relaxation parameter $(\gamma)=100)$ : (a) anode; $(b)$ cathode .

agreement to our past study which showed that a decrease of $\gamma$ (increase of relaxation time) leads to a decrease of $\langle\lambda\rangle$ in anodic catalyst layer and membrane, and an increase of $\langle\lambda\rangle$ in cathodic catalyst layer [10]. Other than the differences in mean values of $\langle\lambda\rangle$, there are no significant effects of $\gamma$ on the response of $\langle\lambda\rangle$ in membrane and catalyst layers. Average vapor concentrations in anode and cathode also exhibit similar response for all values of $\gamma$ except that the response amplitude decreases with a decrease of $\gamma$ (see Figure 7). For example, the response amplitudes of anode and cathode vapor concentrations drop to $0.14 \%$ and $0.12 \%$, respectively, for $\gamma=0.1$ case from $0.23 \%$ for $\gamma=100$. Hence, a decrease of $\gamma$ reduces the sensitivity of vapor concentration to modulating conditions. The fuel cell current density response was found to be insensitive to different relaxation factors (results are not shown here).

\subsection{Effect of modulation frequency}

The effect of modulation frequency was investigated by considering cell voltage modulations with different frequencies. The results presented are for fuel cell, initially operating at a cell voltage of $0.7 \mathrm{~V}$, and subjected to sinusoidal oscillation in cell voltage of $10 \%$ amplitude. Figures 8 and 9 depict the results for the case of $\gamma=100$. The results show, as expected, that the cell water concentration response to imposed oscillation is high at low 


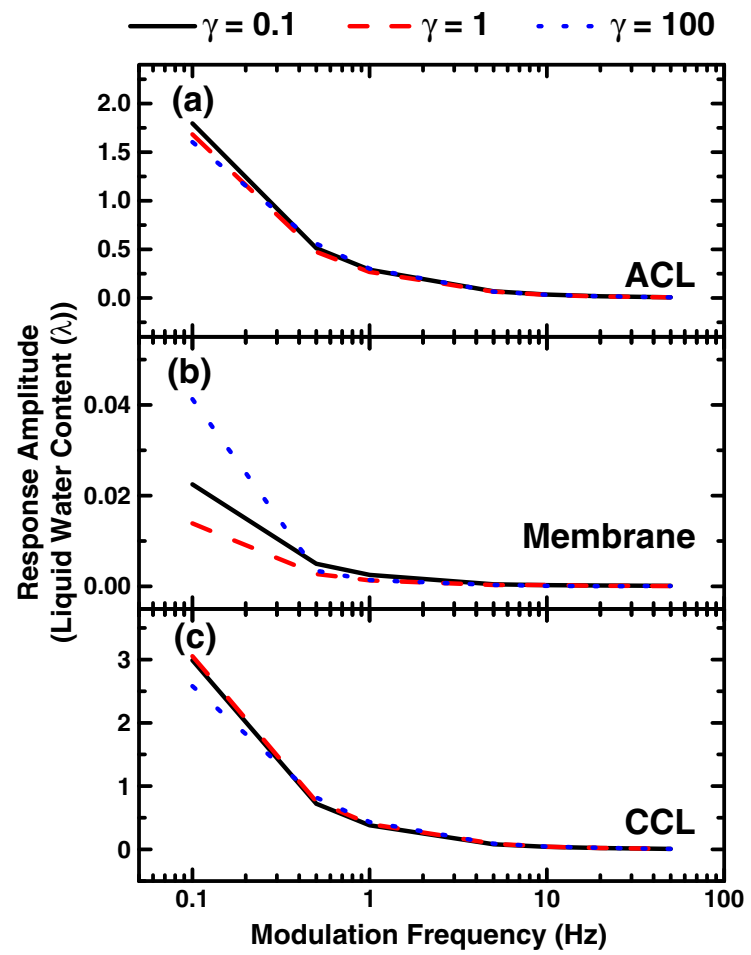

Figure 10. Effect of modulation frequency on the cell average liquid water content response amplitude to modulations in cell voltage for different values of relaxation parameter $(\gamma)$ (modulation amplitude $=10 \%$ ): (a) anodic catalyst layer; (b) cell membrane; (c) cathodic catalyst layer.

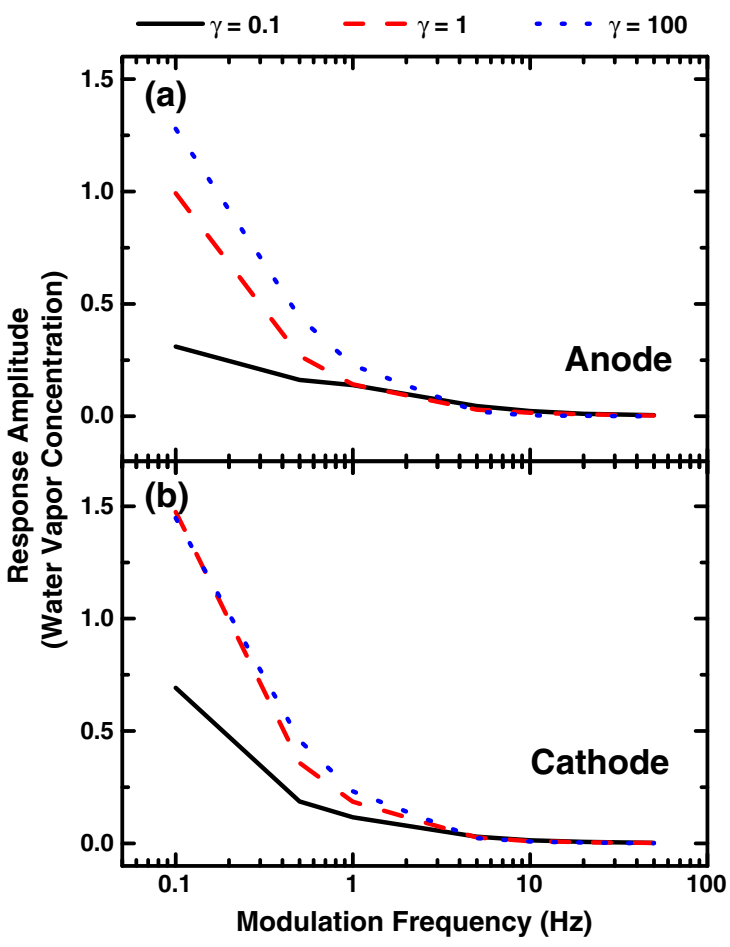

Figure 11. Effect of modulation frequency on the cell water vapor content response amplitude to modulations in cell voltage for different values of relaxation parameter $(\gamma)$ (modulation amplitude $=10 \%$ ): (a) anode; (b) cathode.

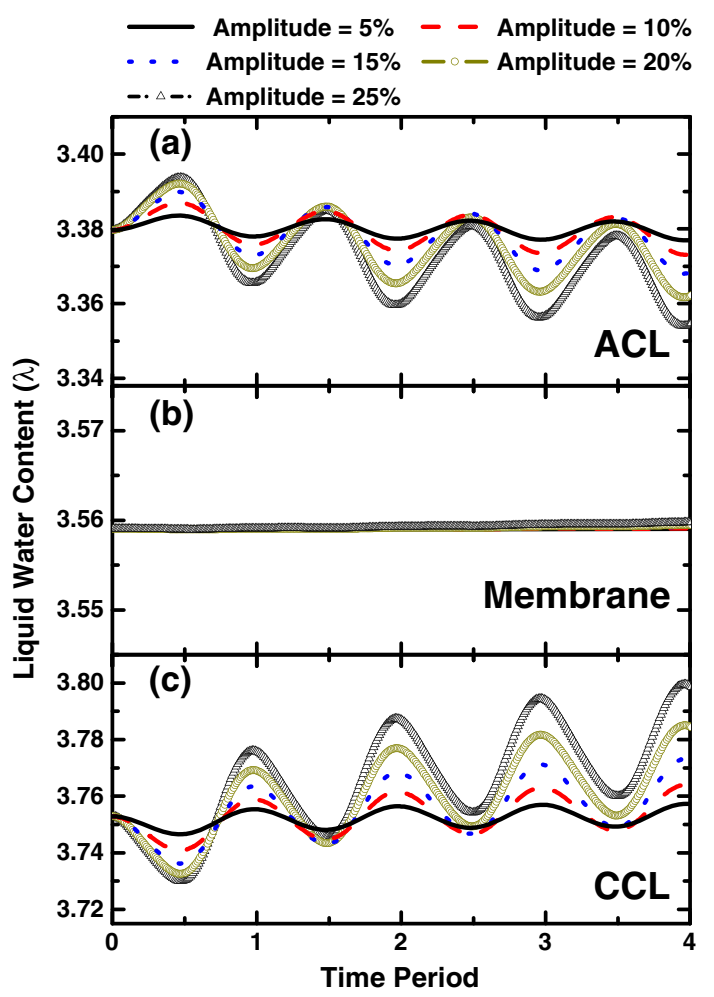

Figure 12. Effect of modulation amplitude on the cell average liquid water content response to modulations in cell voltage (modulation frequency $=1 \mathrm{~Hz}$; relaxation parameter $(\gamma)=100$ ): (a) anodic catalyst layer; (b) cell membrane; (c) cathodic catalyst layer. 


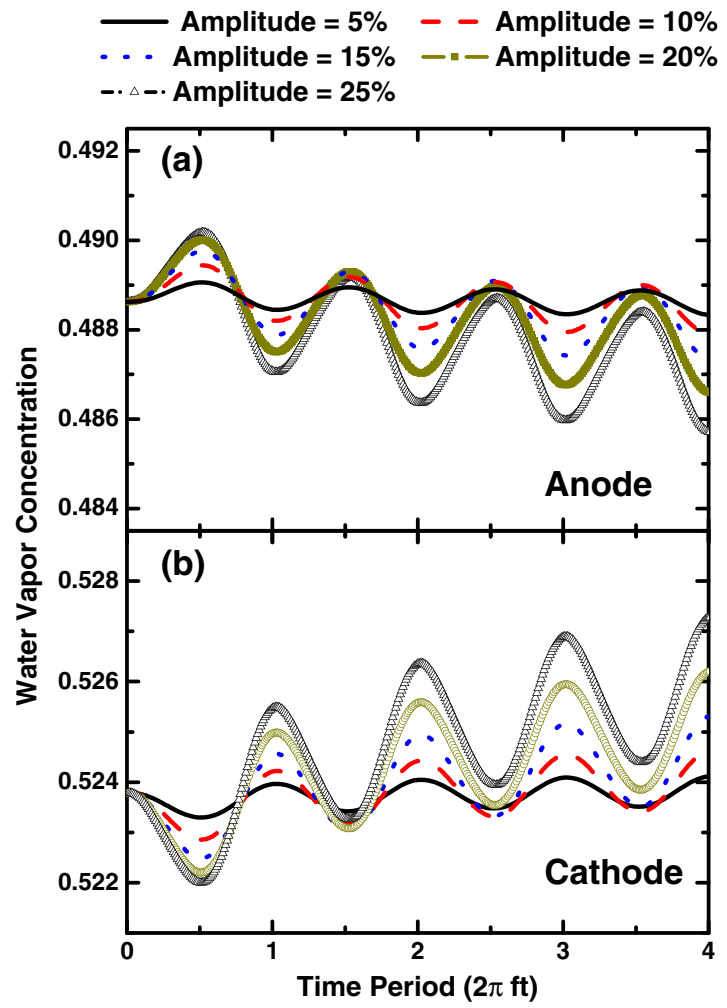

Figure 13. Effect of modulation amplitude on the cell water vapor content response to modulations in cell voltage (modulation frequen$c y=1 \mathrm{~Hz}$; relaxation parameter $(\gamma)=100)$ : (a) anode; (b) cathode

frequencies, and its transient values approach the steadystate values at the corresponding cell voltage values. With an increase of modulation frequencies, the cell response amplitude decreases, and the difference of transient values from the corresponding steady-state values grows. At higher frequencies, the cell's liquid and vapor contents become insensitive to imposed cell voltage modulations. This 'insensitivity' is due to effective neutralization of high frequency fluctuations by diffusion processes over the time period required to convect them. The cut-off frequency corresponding to the cell's insensitivity is approximately $20 \mathrm{~Hz}$. Higher frequencies also increase the initial phase lag in the anode and cathode vapor content response to imposed modulations. Similar results are obtained for other values of $\gamma$ except that the phase lag in vapor content response increases at lower values of $\gamma$ (results are not shown here).

Figures 10 and 11 exhibit the cell's average response amplitude as a function of the imposed modulation frequency for different values of $\gamma$. The results show that the cell response amplitudes are very low for all values of $\gamma$. However, at low frequencies, higher values of $\gamma$ (such as $\gamma=100$ ) bring about a relatively higher cell response to imposed fluctuations than the lower values of $\gamma$. Furthermore, the case of higher value of $\gamma$ also undergoes a relatively sharper decrease in cell response amplitude with

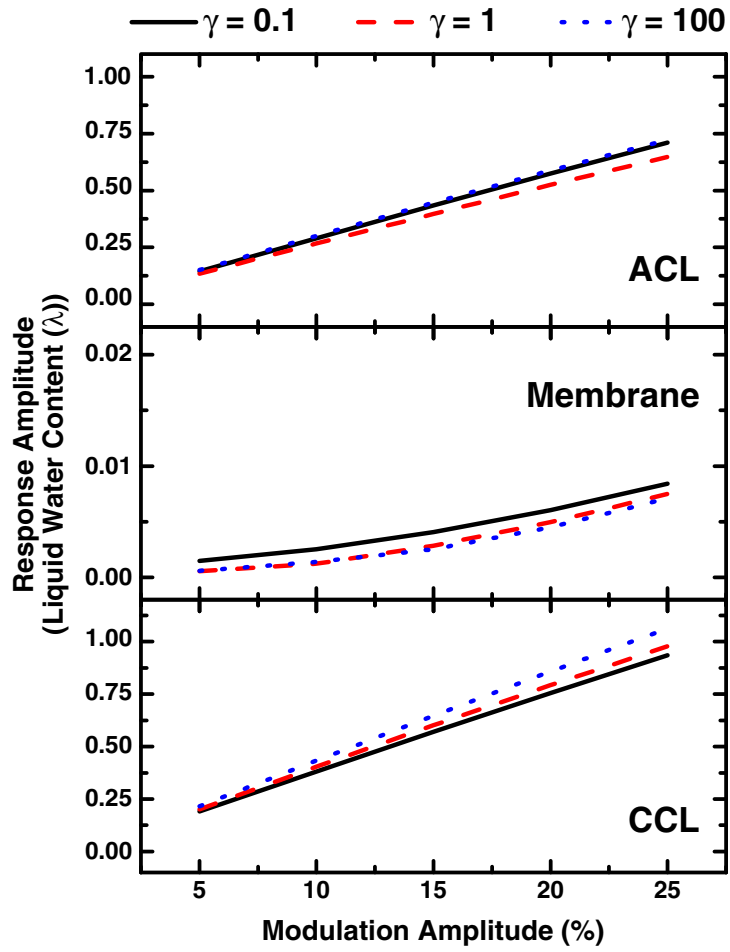

Figure 14. Effect of modulation amplitude on the cell average liquid water content response amplitude to modulations in cell voltage for different values of relaxation parameter $(\gamma)$ (modulation frequency $=1 \mathrm{~Hz}$ ): (a) anodic catalyst layer; (b) cell membrane; (c) cathodic catalyst layer.

the increase of imposed modulation frequency and has a relatively lower cut-off frequency. Compared to liquid water response, the vapor content response is relatively more influenced by $\gamma$.

\subsection{Effect of modulation amplitude}

Figures 12 and 13 show the cell average liquid water and vapor contents as a function of modulation time period for different amplitudes of cell voltage modulations. These results are for fuel cell, initially operating at a cell voltage of $0.7 \mathrm{~V}$ and $\gamma=100$, which is subjected to sinusoidal oscillation in cell voltage of $1 \mathrm{~Hz}$ frequency. The results show that the increase of oscillation amplitude generally increases the cell response. For all modulation amplitudes, the cell response remains sinusoidal, and the response amplitudes are relatively small. For example, for imposed modulation amplitude of $25 \%$, the response amplitudes of average liquid water in anodic and cathodic catalyst layers, and membrane are $0.72 \%, 1.06 \%$, and $0.007 \%$ respectively. The response amplitudes of anode and cathode vapor contents are also very small (approximately $0.55 \%$ for imposed modulation amplitude of $25 \%$ ). For the conditions studied, other than the response amplitude, the modulation amplitude has insignificant influence on any other 


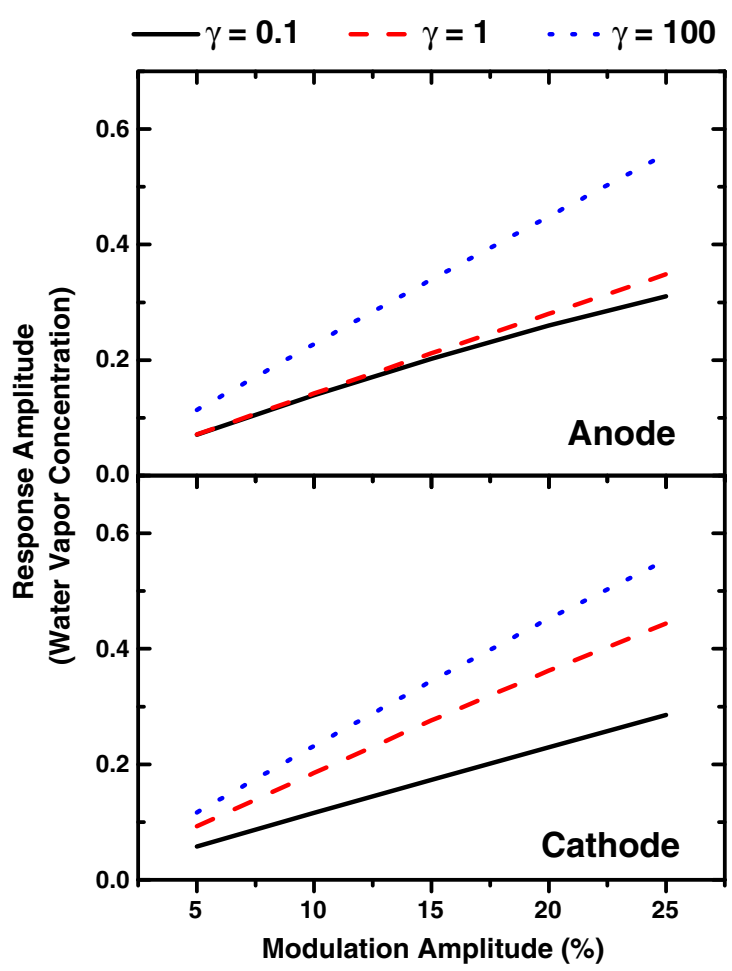

Figure 15. Effect of modulation amplitude on the cell water vapor content response amplitude to modulations in cell voltage for different values of relaxation parameter $(\gamma)$ (modulation frequency $=1 \mathrm{~Hz}$ ): (a) anode; (b) cathode.

aspect of the cell water response. Similar results are obtained for other values of $\gamma$ except that the response amplitudes are lower for lower values of $\gamma$ (results are not shown here).

The effect of modulation amplitude for different values of $\gamma$ is shown in Figures 14 and 15. For all values of $\gamma$, the response amplitudes are low even at higher modulation amplitudes. The response amplitude increases linearly with an increase of modulation amplitude. The response amplitude is higher for higher values of $\gamma$ (i.e., under fast equilibrium condition) except for the membrane liquid water content. The decrease in membrane liquid water sensitivity to imposed changes at higher $\gamma$ was also determined by Vorobev et al. [10]. Compared to liquid water response, the effect of $\gamma$ is higher on water vapor concentration response. The effect of $\gamma$ is more pronounced at higher modulation amplitudes.

\section{CONCLUSIONS}

By employing a numerical model, this study investigated the effect of modulating cell voltage on the water distribution in anode, cathode, catalyst layers, and membrane.
Compared to many other existing models, the present model does not rely on the non-physical assumption of the uptake curve equilibrium between the pore vapor and ionomer water in the catalyst layers. Instead, the transition between the two phases is modeled as a finite-rate equilibration process by employing a phenomenological parameter $\gamma$, which has the physical meaning of the nondimensional reciprocal equilibration time. The study was conducted with a range of $\gamma$ values from 0.1 to 100 , with the higher value of $\gamma$ representing the fast equilibration. The results led to the following conclusions:

- The liquid and vapor concentrations of water in the cell anode, cathode, catalyst layers, and membrane respond to variation in cell voltage. The response is sinusoidal to the sinusoidal imposed modulation. The response amplitude is relatively small, and the water concentration values for the modulating conditions are different from the corresponding steady-state values. In general, a decrease of $\gamma$ reduces the response amplitude (and sensitivity) of vapor concentration to modulating conditions.

- The current density is also strongly influenced by variations in cell voltage. The response is sinusoidal but $180^{\circ}$ out of phase of the imposed sinusoidal modulations. Since the relaxation factor, $\gamma$, is a measure of phase equilibrium process in the catalyst layer, it does not affect the current density response.

- The modulating frequency affects the cell response to the imposed changes. The maximum cell response is observed at low frequencies, where the transient water concentration values for the modulating conditions approach the corresponding steady-state values. With an increase of frequency, the response amplitude decreases, and the cell becomes insensitive to high frequency modulations. The insensitivity occurs at approximately $20 \mathrm{~Hz}$. Higher frequencies also increase the phase lag in the anode and cathode vapor content response to the imposed modulations. The phase lag in response increases at lower values of $\gamma$.

- The response amplitude increases, generally linearly, with an increase of modulation amplitude for all values of $\gamma$. The difference in response amplitudes between different values of $\gamma$ grows at higher modulation amplitude. The increase of modulation amplitude causes a relatively higher change in response amplitude of $\gamma=100$.

\section{NOMENCLATURE}

$a \quad=$ vapor activity

$A \quad=$ modulation amplitude

$C^{k} \quad=$ molar concentration of species $\mathrm{k}\left(\mathrm{mol} / \mathrm{m}^{3}\right)$

$D^{k} \quad=$ diffusivity of species $\mathrm{k}$ in gas mixture $\left(\mathrm{m}^{2} / \mathrm{s}\right)$

$E W \quad=$ equivalent weight of ionomer $(\mathrm{kg} / \mathrm{mol})$

$f \quad=$ modulation frequency $(\mathrm{Hz})$

$F \quad=$ Faraday's constant $(96,487 \mathrm{Col} / \mathrm{mol})$ 


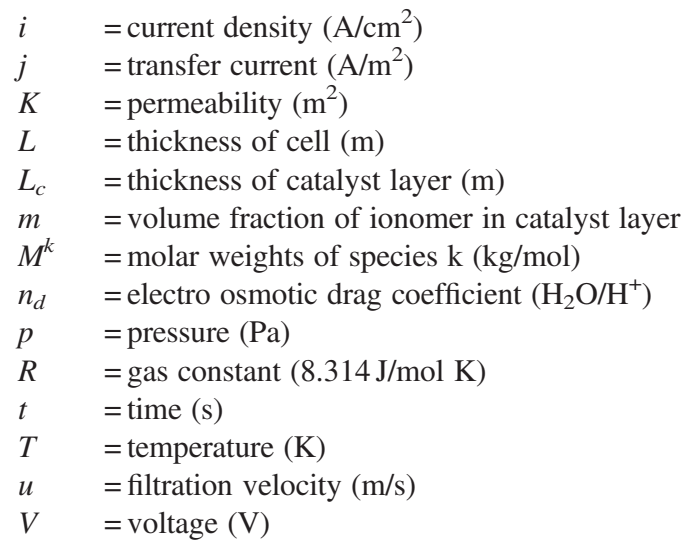

Greek symbols

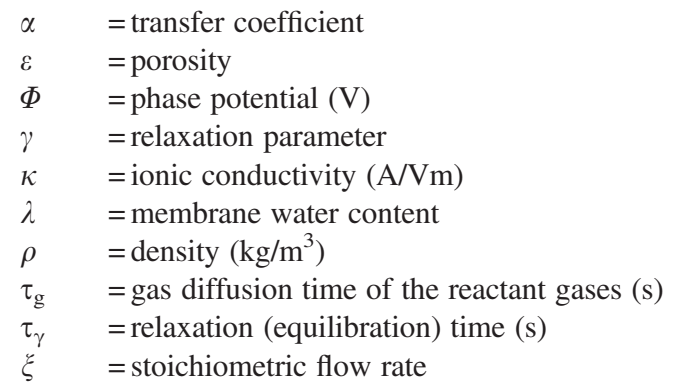

\section{Subscripts and superscripts}

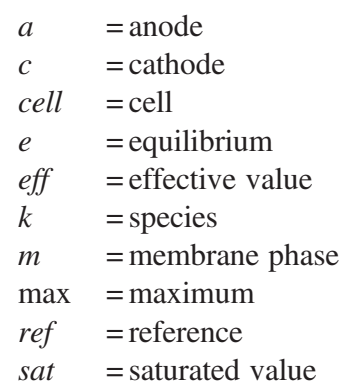

\section{ACKNOWLEDGEMENTS}

The financial support from the HP-Center for Engineering Education and Practice of the University of MichiganDearborn is greatly appreciated.

\section{REFERENCES}

1. Wang C-Y. Fundamental models for fuel cell engineering. Chemical Review 2004; 104:4727-4766.
2. Barbir F, Yazici S. Status and development of PEM fuel cell technology. International Journal of Energy Research 2008; 32:369-378.

3. Zamel N, Li X. A parametric study of multi-phase and multi-species transport in the cathode of PEM fuel cells. International Journal of Energy Research 2008; 32:698-721.

4. Wang Y, Wang C-Y. Transient analysis of polymer electrolyte fuel cells. Electro-chemica Acta 2005; 50:1307-1315.

5. Wang Y, Wang C-Y. Dynamics of polymer electrolyte fuel cells undergoing load changes. Electrochemica Acta 2006; 51:3924-3933.

6. Song D, Wang Q, Liu Z-S, Huang C. Transient analysis for the cathode gas diffusion layer of PEM fuel cells. Journal of Power Sources 2006; 159:928-942.

7. Cho J, Kim H-S, Min K. Transient response of a unit proton-exchange membrane fuel cell under various operating conditions. Journal of Power Sources 2008; 185:118-128.

8. Shimpalee S, Spuckler D, Van Zee JW. Prediction of transient response for a $25-\mathrm{cm}^{2}$ PEM fuel cell. Journal of Power Sources 2007; 167:130-138.

9. Didierjean S, Lottin O, Maranzana G, Geneston T. PEM fuel cell voltage transient response to a thermal perturbation. Electrochemica Acta 2008; 53:7318-7320.

10. Vorobev A, Zikanov O, Shamim T. A computational model of a PEM fuel cell with finite vapor absorption rate. Journal of Power Sources 2007; 166:92-103.

11. Wang Y, Wang C-Y. Two-phase transients of polymer electrolyte fuel cells. Journal of the Electrochemical Society 2007; 154:B636-B646.

12. Hussaini IS, Wang C-Y. Transients of water distribution and transport in PEM fuel cells. Journal of the Electrochemical Society 2009; 156:B1394-B1400.

13. Zhang Z, Jia L, Wang X, Ba L. Effects of inlet humidification on PEM fuel cell dynamic behaviours. International Journal of Energy Research 2011; 35:376-388.

14. Berg P, Promislow K, St-Pierre J, Stumper J, Wetton B. Water management in PEM fuel cells. Journal of the Electrochemical Society 2004; 151:A341-A353.

15. Berg P, Novruzi A, Promislow K. Analysis of a cathode catalyst layer model for a polymer electrolyte fuel cell. Chemical Engineering Science 2006; 61:4316-4331.

16. Wang Y, Wang C-Y. Modeling polymer electrolyte fuel cells with large density and velocity changes. Journal of the Electrochemical Society 2005; 152: A445-A453. 
17. Hu G, Fan JR. Transient computation fluid dynamics modeling of a single proton exchange membrane fuel cell with serpentine channel. Journal of Power Sources 2007; 165:171-184.

18. Fardadi M, Mueller F, Jabbari F. Feedback control of solid oxide fuel cell spatial temperature variation. Journal of Power Sources 2010; 195:4222-4233.
19. Haddad A, Bouyekhf R, El Moudni A. Dynamic modeling and water management in proton exchange membrane fuel cell. International Journal of Hydrogen Energy 2008; 33:6239-6252.

20. Liu Z, Mao Z, Wang C, Zhuge W, Zhang Y. Numerical simulation of a mini PEMFC stack. Journal of Power Sources 2006; 160:1111-1121. 